\title{
A FIXED POINT THEOREM FOR A PAIR OF MAPS \\ SATISFYING A GENERAL CONTRACTIVE \\ CONDITION OF INTEGRAL TYPE
}

P. VIJAYARAJU, B. E. RHOADES, AND R. MOHANRAJ

Received 18 October 2004 and in revised form 20 July 2005

We give a general condition which enables one to easily establish fixed point theorems for a pair of maps satisfying a contractive inequality of integral type.

Branciari [1] obtained a fixed point result for a single mapping satisfying an analogue of Banach's contraction principle for an integral-type inequality. The second author [3] proved two fixed point theorems involving more general contractive conditions. In this paper, we establish a general principle, which makes it possible to prove many fixed point theorems for a pair of maps of integral type.

Define $\Phi=\left\{\varphi: \varphi: \mathbb{R}^{+} \rightarrow \mathbb{R}\right\}$ such that $\varphi$ is nonnegative, Lebesgue integrable, and satisfies

$$
\int_{0}^{\epsilon} \varphi(t) d t>0 \quad \text { for each } \epsilon>0
$$

Let $\psi: \mathbb{R}^{+} \rightarrow \mathbb{R}^{+}$satisfy that

(i) $\psi$ is nonnegative and nondecreasing on $\mathbb{R}^{+}$,

(ii) $\psi(t)<t$ for each $t>0$,

(iii) $\sum_{n=1}^{\infty} \psi^{n}(t)<\infty$ for each fixed $t>0$.

Define $\Psi=\{\psi: \psi$ satisfies (i) $-($ iii) $\}$.

Lemma 1. Let $S$ and $T$ be self-maps of a metric space $(X, d)$. Suppose that there exists a sequence $\left\{x_{n}\right\} \subset X$ with $x_{0} \in X, x_{2 n+1}:=S x_{2 n}, x_{2 n+2}:=T x_{2 n+1}$, such that $\overline{\left\{x_{n}\right\}}$ is complete and there exists $a k \in[0,1)$ such that

$$
\int_{0}^{d(S x, T y)} \varphi(t) d t \leq \psi\left(\int_{0}^{d(x, y)} \varphi(t) d t\right)
$$

for each distinct $x, y \in \overline{\left\{x_{n}\right\}}$ satisfying either $x=$ Ty or $y=S x$, where $\varphi \in \Phi, \psi \in \Psi$. 
2360 Fixed point theorem for a pair of maps

Then, either

(a) Sor Thas a fixed point in $\left\{x_{n}\right\}$ or

(b) $\left\{x_{n}\right\}$ converges to some point $p \in X$ and

$$
\int_{0}^{d\left(x_{n}, p\right)} \varphi(t) d t \leq \sum_{i=n}^{\infty} \psi^{i}(d) \quad \text { for } n>0,
$$

where

$$
d:=\int_{0}^{d\left(x_{0}, x_{1}\right)} \varphi(t) d t
$$

Proof. Suppose that $x_{2 n+1}=x_{2 n}$ for some $n$. Then $x_{2 n}=x_{2 n+1}=S x_{2 n}$, and $x_{2 n}$ is a fixed point of $S$. Similarly, if $x_{2 n+2}=x_{2 n+1}$ for some $n$, then $x_{2 n+1}$ is a fixed point of $T$.

Now assume that $x_{n} \neq x_{n+1}$ for each $n$. With $x=x_{2 n}, y=x_{2 n+1}$, (2) becomes

$$
\int_{0}^{d\left(x_{2 n+1}, x_{2 n+2}\right)} \varphi(t) d t \leq \psi\left(\int_{0}^{d\left(x_{2 n}, x_{2 n+1}\right)} \varphi(t) d t\right) .
$$

Substituting $x=x_{2 n}, y=x_{2 n-1},(2)$ becomes

$$
\int_{0}^{d\left(x_{2 n+1}, x_{2 n}\right)} \varphi(t) d t \leq \psi\left(\int_{0}^{d\left(x_{2 n}, x_{2 n-1}\right)} \varphi(t) d t\right) .
$$

Therefore, for each $n \geq 0$,

$$
\int_{0}^{d\left(x_{n}, x_{n+1}\right)} \varphi(t) d t \leq \psi\left(\int_{0}^{d\left(x_{n-1}, x_{n}\right)} \varphi(t) d t\right) \leq \cdots \leq \psi^{n}(d) .
$$

Let $m, n \in \mathbb{N}, m>n$. Then, using the triangular inequality,

$$
d\left(x_{n}, x_{m}\right) \leq \sum_{i=n}^{m-1} d\left(x_{i}, x_{i+1}\right)
$$

It can be shown by induction that

$$
\int_{0}^{d\left(x_{n}, x_{m}\right)} \varphi(t) d t \leq \sum_{i=n}^{m-1} \int_{0}^{d\left(x_{i}, x_{i+1}\right)} \varphi(t) d t
$$

Using (7) and (9),

$$
\int_{0}^{d\left(x_{n}, x_{m}\right)} \varphi(t) d t \leq \sum_{i=n}^{\infty} \psi^{i}(d) \leq \sum_{i=n}^{\infty} \psi^{i}(d) .
$$

Taking the limit of (10) as $m, n \rightarrow \infty$ and using condition (iii) for $\psi$, it follows that $\left\{x_{n}\right\}$ is Cauchy, hence convergent, since $X$ is complete. Call the limit $p$. Taking the limit of (10) as $m \rightarrow \infty$ yields (3). 
Theorem 2. Let $(X, d)$ be a complete metric space, and let $S$, $T$ be self-maps of $X$ such that for each distinct $x, y \in X$,

$$
\int_{0}^{d(S x, T y)} \varphi(t) d t \leq \psi\left(\int_{0}^{M(x, y)} \varphi(t) d t\right),
$$

where $k \in[0,1), \varphi \in \Phi, \psi \in \Psi$, and

$$
M(x, y):=\max \left\{d(x, y), d(x, S x), d(y, T y), \frac{[d(x, T y)+d(y, S x)]}{2}\right\} .
$$

Then $S$ and T have a unique common fixed point.

Proof. We will first show that any fixed point of $S$ is also a fixed point of $T$, and conversely.

Let $p=S p$. Then

$$
M(p, p)=\max \left\{0,0, d(p, T p), \frac{d(p, T p)}{2}\right\}=d(p, T p),
$$

and (11) becomes

$$
\int_{0}^{d(p, T p)} \varphi(t) d t \leq \psi\left(\int_{0}^{d(p, T p)} \varphi(t) d t\right),
$$

which, from (1), implies that $p=T p$.

Similarly, $p=T p$ implies that $p=S p$.

We will now show that $S$ and $T$ satisfy (2).

$$
M(x, S x)=\max \left\{d(x, S x), d(x, S x), d(S x, T S x), \frac{[d(x, T S x)+0]}{2}\right\} .
$$

From the triangular inequality,

$$
\frac{d(x, T S x)}{2} \leq \frac{[d(x, S x)+d(S x, T S x)]}{2} \leq \max \{d(x, S x), d(S x, T S x)\} .
$$

Thus, (11) becomes

$$
\int_{0}^{d(S x, T S x)} \varphi(t) d t \leq k \int_{0}^{d(S x, T S x)} \varphi(t) d t
$$

a contradiction to (1).

Therefore, for all $x \in X, M(x, S x)=d(x, S x)$, and (2) is satisfied. If condition (a) of Lemma 1 is true, then $S$ or $T$ has a fixed point. But it has already been shown that any fixed point of $S$ is also a fixed point of $T$, and conversely. Thus $S$ and $T$ have a common fixed point.

Suppose that conclusion (b) of Lemma 1 is true. Then, from (3),

$$
\int_{0}^{d\left(S x_{2 n}, T p\right)} \varphi(t) d t \leq \psi\left(\int_{0}^{d\left(x_{2 n}, p\right)} \varphi(t) d t\right),
$$

which implies, since $X$ is complete, that $\lim d\left(S x_{2 n}, T p\right)=0$. 
2362 Fixed point theorem for a pair of maps

Therefore,

$$
d(p, T p) \leq d\left(p, S x_{2 n}\right)+d\left(S x_{2 n}, T p\right) \longrightarrow 0,
$$

and $p$ is a fixed point of $T$, hence a fixed point of $S$. Condition (11) clearly implies uniqueness of the fixed point.

Every contractive condition of integral type automatically includes a corresponding contractive condition not involving integrals, by setting $\varphi(t) \equiv 1$ over $\mathbb{R}^{+}$.

There are many contractive conditions of integral type which satisfy (2). Included among these are the analogues of the many contractive conditions involving rational expressions and/or products of distances. We conclude this paper with one such example.

Corollary 3. Let $(X, d)$ be a complete metric space, $S$ and $T$ self-maps of $X$ such that, for each distinct $x, y \in X$,

$$
\int_{0}^{d(S x, T y)} \varphi(t) d t \leq k \int_{0}^{n(x, y)} \varphi(t) d t
$$

where $\varphi \in \Phi, k \in[0,1)$, and

$$
n(x, y):=\max \left\{\frac{d(y, T y)[1+d(x, S x)]}{1+d(x, y)}, d(x, y)\right\} .
$$

Then $S$ and T have a unique common fixed point.

Proof.

$$
n(x, S x)=\max \{d(S x, T S x), d(x, S x)\} .
$$

As in the proof of Theorem 2, it is easy to show that any fixed point of $S$ is also a fixed point of $T$, and conversely.

If $n(x, S x)=d(S x, T S x)$, then an argument similar to that of Theorem 2 leads to a contradiction. Therefore $n(x, S x)=d(x, S x)$, and either $S$ or $T$ has a common fixed point, or (3) is satisfied. In the latter case, with $\lim x_{n}=p, n(p, p)=0$, so that, from (20), $p$ is a fixed point of $S$, hence of $T$. Uniqueness of $p$ is easily established.

Corollary 3 is also a consequence of Lemma 1.

We now provide an example, kindly supplied by one of the referees, to show that Lemma 1 is more general than [2, Theorem 3.1].

Example 4. Let $X:=\{1 / n: n \in \mathbb{N} \cup\{0\}\}$ with the Euclidean metric and $S$, $T$ are self-maps of $X$ defined by

$$
S\left(\frac{1}{n}\right)=\left\{\begin{array}{ll}
\frac{1}{n+1} & \text { if } n \text { is odd, } \\
\frac{1}{n+2} & \text { if } n \text { is even, } \\
0 & \text { if } n=\infty,
\end{array} \quad T\left(\frac{1}{n}\right)= \begin{cases}\frac{1}{n+1} & \text { if } n \text { is even, } \\
\frac{1}{n+2} & \text { if } n \text { is odd } \\
0 & \text { if } n=\infty .\end{cases}\right.
$$


For each $n$, define $x_{2 n+1}=S x_{2 n}, x_{2 n+2}=T x_{2 n+1}$. With $x_{0}=1$, let $O(1)$ denote the orbit of $x_{0}=1$; that is, $O(1)=\{1,1 / 2,1 / 3, \ldots\}$ and $\overline{O(1)}=O(1) \cup\{0\}=X$. For $x, y \in O(1)$, $y=1 / m, m$ even and $x=1 / n=T y=1 /(m+1), S x=1 /(m+2)$, so that

$$
\begin{gathered}
d(S x, T y)=\left|\frac{1}{m+1}-\frac{1}{m+1}\right|=\frac{1}{m+1}-\frac{1}{m+2}=\frac{1}{(m+1)(m+2)}, \\
d(x, y)=\left|\frac{1}{n}-\frac{1}{m}\right|=\left|\frac{1}{m+1}-\frac{1}{n}\right|=\frac{1}{m}-\frac{1}{m+1}=\frac{1}{m(m+1)} .
\end{gathered}
$$

Thus

$$
\frac{d(S x, T y)}{d(x, y)}=\frac{m}{m+2} \leq 1
$$

Also

$$
\sup _{n \in \mathbb{N}} \frac{d(S x, T y)}{d(x, y)}=1
$$

so that there is no number $c \in[0,1)$ such that $d(S x, T y) \leq c d(x, y)$ for $x, y \in O(1)$ and $x=T y$. Therefore, [2, Theorem 3.1] cannot be used. On the other hand, the hypotheses of Lemma 1 are satisfied. To see this, it will be shown that condition (2) is satisfied for some $\varphi \in \Phi$.

We will first show that for any $x=1 / n, y=1 / m \in O(1)$ satisfying either $x=T y$ or $y=S x$,

$$
d(S x, T y) \leq\left|\frac{1}{n+1}-\frac{1}{m+1}\right|
$$

There are four cases.

Case 1. $y=1 / m, m$ even, $x=1 / n=T y=1 /(m+1)$, and $S x=1 /(m+2)$. Then

$$
d(S x, T y)=\left|\frac{1}{m+2}-\frac{1}{m+1}\right|=\left|\frac{1}{n+1}-\frac{1}{m+1}\right| .
$$

Case 2. $y=1 / m, m$ odd, $x=1 / n=T y=1 /(m+2)$, and $S x=1 /(m+3)$. Then

$$
\begin{aligned}
d(S x, T y) & =\left|\frac{1}{m+3}-\frac{1}{m+2}\right|=\frac{1}{m+2}-\frac{1}{m+3} \\
& \leq \frac{1}{m+1}-\frac{1}{m+3}=\left|\frac{1}{n+1}-\frac{1}{m+1}\right| .
\end{aligned}
$$

Case 3. $x=1 / n, n$ even, $y=1 / m=S x=1 /(n+2)$, and $T y=1 /(n+3)$. Then

$$
\begin{aligned}
d(S x, T y) & =\left|\frac{1}{n+2}-\frac{1}{n+3}\right|=\frac{1}{n+2}-\frac{1}{n+3} \\
& \leq \frac{1}{n+1}-\frac{1}{n+3}=\left|\frac{1}{n+1}-\frac{1}{n+3}\right|
\end{aligned}
$$


Case 4. $x=1 / n, n$ odd, $y=1 / m=S x=1 /(n+1)$, and $T y=1 /(n+2)$. Then

$$
d(S x, T y)=\left|\frac{1}{n+1}-\frac{1}{n+2}\right|=\left|\frac{1}{n+1}-\frac{1}{m+1}\right| .
$$

Thus in all cases, (20) is satisfied.

Define $\varphi$ by $\varphi(t)=t^{1 / 2-2}[1-\log t]$ for $t>0$ and $\varphi(0)=0$. Then, for any $\tau>0$,

$$
\int_{0}^{\tau} \varphi(t) d t=\tau^{1 / \tau}
$$

and $\varphi \in \Phi$.

Using [1, Example 3.6],

$$
\begin{aligned}
\int_{0}^{d(S x, T y)} \varphi(t) d t & \leq d(S x, T y)^{1 / d(S x, T y)} \\
& \leq\left|\frac{1}{n+1}-\frac{1}{m+1}\right|^{1 /|(1 / n+1)-(1 / m+1)|} \\
& \leq \frac{1}{2}\left|\frac{1}{n}-\frac{1}{m}\right|^{1 /|(1 / n)-(1 / m)|}=d(x, y)^{1 / d(x, y)}
\end{aligned}
$$

for each $x, y$ as in Lemma 1 , and condition (2) is satisfied with $\psi(t)=t / 2$.

\section{Acknowledgment}

The authors thank each of the referees for careful reading of the manuscript.

\section{References}

[1] A. Branciari, A fixed point theorem for mappings satisfying a general contractive condition of integral type, Int. J. Math. Math. Sci. 29 (2002), no. 9, 531-536.

[2] S. Park, Fixed points and periodic points of contractive pairs of maps, Proc. College Natur. Sci. Seoul Nat. Univ. 5 (1980), no. 1, 9-22.

[3] B. E. Rhoades, Two fixed-point theorems for mappings satisfying a general contractive condition of integral type, Int. J. Math. Math. Sci. 2003 (2003), no. 63, 4007-4013.

P. Vijayaraju: Department of Mathematics, Anna University, Chennai-600 025, India

E-mail address: vijay@annauniv.edu

B. E. Rhoades: Department of Mathematics, Indiana University, Bloomington, IN 47405-7106, USA

E-mail address: rhoades@indiana.edu

R. Mohanraj: Department of Mathematics, Anna University, Chennai-600 025, India

E-mail address: vrmraj@yahoo.com 


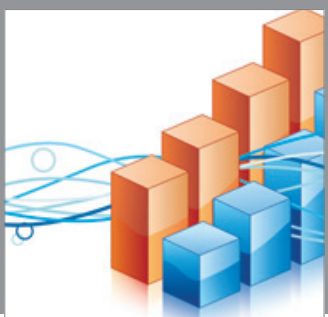

Advances in

Operations Research

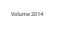

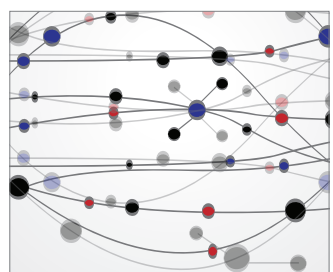

\section{The Scientific} World Journal
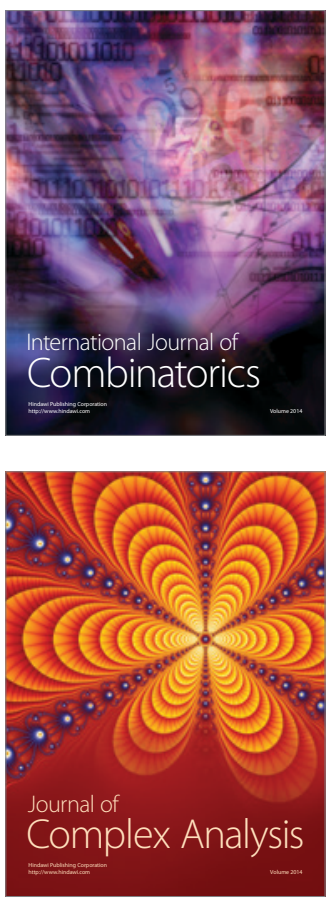

International Journal of

Mathematics and

Mathematical

Sciences
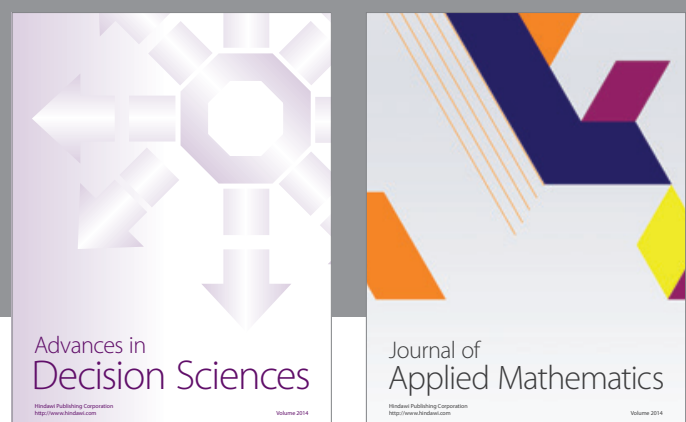

Journal of

Applied Mathematics
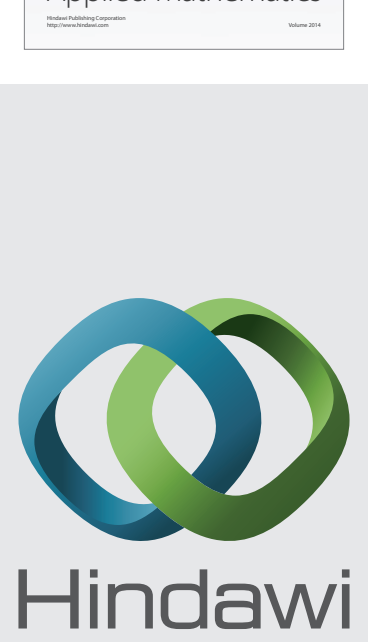

Submit your manuscripts at http://www.hindawi.com
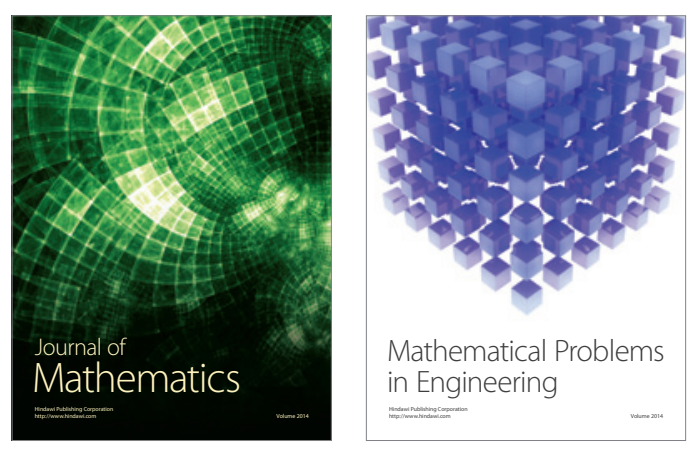

Mathematical Problems in Engineering
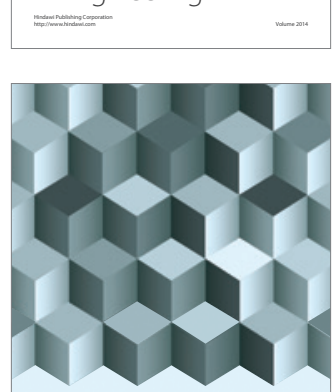

Journal of

Function Spaces
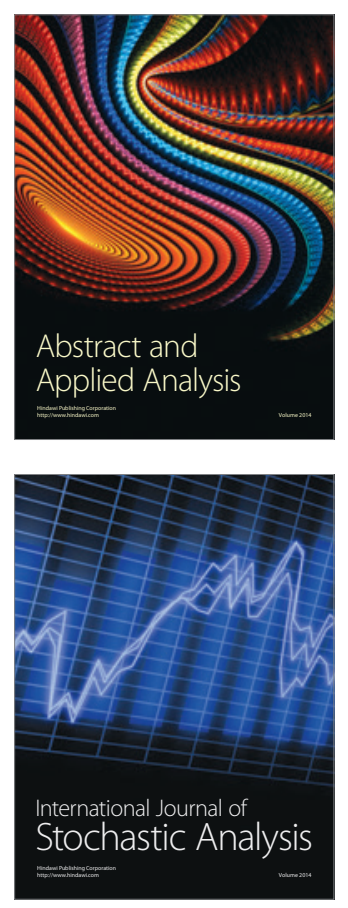

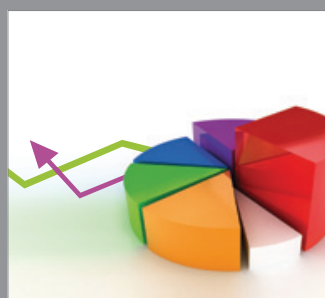

ournal of

Probability and Statistics

Promensencen
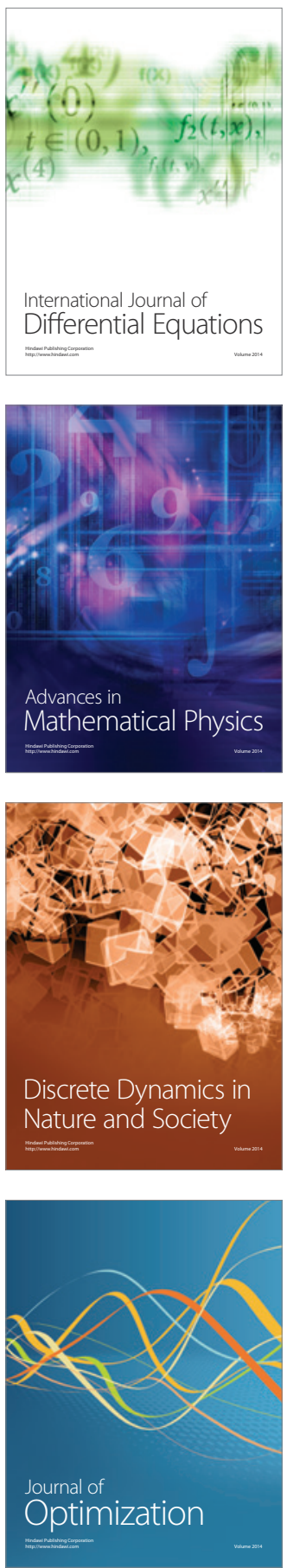